\title{
Highly resistant multicoloring with 3 attackers and 1 malfunctioning vertex
}

\author{
Tanja Vojković; Damir Vukičević \\ Department of Mathematics, Faculty of Science, \\ Split, Croatia
}

\begin{abstract}
In this paper we explore a way of securing a secret inside a graph by observing pieces of the secret as colors assigned to the graph vertices. If a graph allows a highly $(a, b)$-resistant $k$-multicoloring then a secret can be divided into $k$ parts and sets of those parts distributed to the vertices of the graph so that no $a$ attackers can steal the secret, and when $a$ attackers and $b$ malfuntioning vertices leave the graph, the secret is still whole in the remaining graph. In this paper we explore how many vertices a graph must have in order to allow a highly $(3,1)$-resistant $k$-multicoloring, and what is the minimal number of colors, for graphs that do allow such multicoloring.
\end{abstract}

Keywords: graph theory, graph coloring, multicoloring, secret sharing

MSC: 05C82, 05C15, 68R10, 94A62

\section{Introduction}

In paper Multicoloring of graphs to secure a secret, [7], we were motivated by a problem of securing a secret by dividing it into parts and distributing them to the participants of some network. This is a known method of securing a secret [6]. In our model, there are $a$ attacker vertices in the network, trying to read the secret or disable the group from reading it. Here we make an additional assumption,

\footnotetext{
*Corresponding author: tanja@pmfst.hr
}

DOI: https://doi.org/10.5592/CO/CCD.2018.12 
that besides the $a$ attacker vertices, there are $b$ vertices that are malfunctioning and leaving the network. The secret is secure if $a$ attackers didn't steal the secret and if the group is still able to read the whole secret after $a$ attacker vertices, their neighbors, and $b$ malfunctioning vertices are removed from the network. We represented the network with graph and parts of the secret with colors assigned to the vertices. Coloring and multicoloring of graphs are often used to model some real-life problem, like scheduling or frequency allocation and there are many new colorings defined with different coloring conditions $[2,3,4,5,9]$. This prompted us to define a highly $(a, b)$-resistant multicoloring with the conditions that make the secret secure.

Our goal is to analyze minimal number of vertices a graph must have to allow a highly $(a, b)$-resistant multicoloring, for given $a$ and $b$, and if such a coloring exists for a graph $G$, to determine what is the minimal number of colors. The results for $a=1, b \in \mathbb{N}$ and $a=2, b=1,2,3$ are presented in paper Highly $(a, b)$-resistant multicoloring of graphs, [8], which is not yet published at this point. It can be obtained from the authors by request, however, it is not necessary for understanding this paper.

In this manuscript we analyze what graphs will allow a highly $(a, b)$-resistant multicoloring for $a=3$ and $b=1$.

\section{Preliminaries}

We will mostly use standard definitions and notations of graph theory from [1], and the rest we present in this section. For graph $G$ and $u \in V(G)$, with $N(u)=N_{G}(u)$ we denote the set of neighbors of $u$ in $G$, and $M(u)=M_{G}(u)=N(u) \cup\{u\}$. Naturally, for $A \subseteq V(G)$ we denote:

$$
\begin{aligned}
& N(A)=N_{G}(A)=\bigcup_{u \in A} N_{G}(u) ; \\
& M(A)=M_{G}(A)=\bigcup_{u \in A} M_{G}(u) .
\end{aligned}
$$

First, let us give the formal definition of highly $(a, b)$-resistant multicoloring.

Definition 1. Let $G$ be a graph, and $a, b, k \in \mathbb{N}_{0}$. Vertex $k$-multicoloring $\kappa$ of $G$ is called a highly $(a, b)$-resistant vertex $k$-multicoloring if for each $A, B \subseteq V(G)$, where $|A|=a$ and $|B|=b$, the following holds: 
1. There exists a component $H$ of the graph $G \backslash\left(M_{G}(A) \cup B\right)$ such that

$$
\bigcup_{u \in V(H)} \kappa(u)=\{1, \ldots, k\} .
$$

2. $\bigcup_{u \in A} \kappa(u) \neq\{1, \ldots, k\}$.

We will say that graph $G$ allows a highly $(a, b)$-resistant multicoloring if a multicoloring function $\kappa$ exists that is highly $(a, b)$-resistant. We will denote by $H R_{a, b}(n)=k$ the fact that there exists a graph $G$ with $n$ vertices that allows a highly $(a, b)$-resistant $k$-multicoloring, where $k$ is the minimal number of colors needed.

It can be easily proven that if a graph $G$ with $n$ vertices allows a highly $(a, b)$ resistant $k$-multicoloring than the same graph allows a highly $(a, b)$-resistant $(k+1)$ multicoloring, and there exists a graph $G^{\prime}$ with $n+1$ vertices that allows a highly $(a, b)$-resistant $k$-multicoloring.

In our proofs, we will make use of the notion of an $l$-separable graph.

Definition 2. Let $G$ be a graph and $a, b, l \in \mathbb{N}_{0}$. We will say that $G$ is l-separable with $(a, b)$ vertices, if subsets $A, B \subseteq V(G)$ exist, with $|A|=a$ and $|B|=b$, such that all the components in graph $G \backslash\left(M_{G}(A) \cup B\right)$ have at most $l$ vertices.

For a graph to allow a highly $(3,1)$-resistant multicoloring, no 3 vertices can have all the colors, so we will need the notion of a 3 -separable graph.

\section{Highly (3,1)-resistant multicoloring}

Our main theorem answers two questions:

1. What is the minimal number of vertices a graph must have in order to allow a highly $(3,1)$-resistant multicoloring?

2 . What is the minimal number of colors needed for a highly $(3,1)$-resistant multicoloring in all the graphs that allow such a coloring?

In order to shorten the proof of the theorem, we will first prove several lemmas.

Lemma 1. Let $G$ be a graph.

i) If $G$ has at most 8 vertices it is 3 -separable with $(1,1)$ vertices.

ii) If $G$ has at most 10 vertices it is 3 -separable with $(2,0)$ vertices.

iii) If $G$ has at most 12 vertices it is 3 -separable with $(2,1)$ vertices.

iv) If $G$ has at most 13 vertices it is 3 -separable with $(3,0)$ vertices. 
Proof. All the claims will be proven for connected graphs, and from that it easily follows that they also hold for disconnected graphs.

i) Let $G$ be a connected graph with at most 8 vertices. If $G$ contains a vertex $u$ of degree at least 3 the claim is obvious, and if that is not the case then let $u$ be any vertex of degree 2 in $G$. $G \backslash M(u)$ has at most 5 vertices and it is a union of paths so it is 3 -separable with $(0,1)$ vertices.

ii) Let $G$ be a connected graph with at most 10 vertices. If there exists a vertex $u$ of degree at least 3 in $G$ then $G \backslash M(u)$ has at most 6 vertices. If there exists a vertex of degree at least 2 in $G \backslash M(u)$, then $G \backslash M(u)$ is 3 -separable with $(1,0)$ vertices, and if all the vertices have the degree at most 1 then all the components already have at most 3 vertices. On the other hand, if all the vertices in $G$ have the degree at most 2 , and $u$ is any vertex of degree 2 then $G \backslash M(u)$ has at most 7 vertices and it is a union of paths so it is easily seen that it is 3- separable with $(1,0)$ vertices.

iii) Let $G$ be a connected graph with at most 12 vertices. If there exists a vertex $u$ of degree at least 3 in $G$ then $G \backslash M(u)$ has at most 8 vertices and the claim now follows from i). On the other hand, if the highest degree in $G$ is at most 2 then by observing any vertex $u$ of degree $2, G \backslash M(u)$ remains with at most 9 vertices and it is a union of paths so the claim again easily follows.

iv) This case is proven in paper [7].

Lemma 2. i) A graph $G$ with 9 vertices, $\Delta(G) \leq 3$ and $\delta(G)=1$ is 3-separable with $(1,1)$ vertices.

ii) A graph $G$ with 13 vertices, $\Delta(G) \leq 3$ and $\delta(G)=1$ is 3 -separable with $(2,1)$ vertices.

Proof. i) Let $G$ be a connected graph with 9 vertices, $\Delta(G) \leq 3$ and $\delta(G)=1$. Let $x$ be a vertex of degree 1 in $G$, and let us denote its only neighbor by $y$. If $y$ has the degree 2 then let us denote the other neighbor of $y$ by $w . G \backslash\{w\}$ has 8 vertices and at most 6 vertices in its largest component. If we denote any vertex $u$ of degree at least 2 in that component then $G \backslash(M(u) \cup\{w\})$ is a graph with all components of size at most 3. On the other hand, if $y$ has the degree 3 , let us denote its other 2 neighbors by $w_{1}$ and $w_{2}$. At least one of them has another neighbor, not in $\left\{x, y, w_{1}, w_{2}\right\}$. Let us assume that $w_{1}$ has another neighbor and let us denote it by $u$. If $u$ has the degree 3 than $G \backslash M(u)$ has 5 vertices and either its largest component has at most 4 vertices (if $u$ was adjacent to $w_{2}$ ), so it is 3 -separable with $(0,1)$ vertices, or its largest component has at most 5 vertices and by removing $w_{2}$ obtain a graph with all components of size at most 3 . If $u$ has the degree 2 , then either it is adjacent 
to $w_{2}$ and then by removing the third neighbor of $w_{2}$ and its neighbors, and $w_{1}$, we obtain a graph with components of size at most 3, or $G \backslash\left(M(u) \cup\left\{w_{2}\right\}\right)$ is such a graph. If $G$ is disconnected it can be easily seen that the claim also stands.

ii) Let $G$ be a connected graph with 13 vertices, $\Delta(G) \leq 3$ and $\delta(G)=1$. Let $x$ be a vertex of degree 1 , and $y$ its only neighbor. If $y$ has the degree 2 let us denote by $w$ its other neighbor. $G \backslash\{w\}$ has 1 component of 2 vertices, $x$ and $y$, and the remaining graph has 10 so it is 3 -separable with $(2,0)$ vertices by Lemma 1 ii). If $y$ has the degree 3 let us denote its other 2 neighbors by $w_{1}$ and $w_{2}$. Now, if there exists a vertex $u$ in $G \backslash\{y\}$ of degree 3 in $G$ then $G \backslash M(u)$ has 9 vertices, $\Delta(G \backslash M(u)) \leq 3$ and $\delta(G \backslash M(u)) \leq 1$, so the claim follows from i) (if $\delta(G \backslash M(u))=1$ ) or from Lemma 1 i) (if $\delta(G \backslash M(u))=0$ ). If this is not the case then the only vertex with degree 3 in $G$ is $y$ and $G \backslash M(y)$ has 9 vertices in a union of paths and it is easy to see that the claim also follows. If $G$ is disconnected the claim also holds.

Lemma 3. Let $G$ be a graph with 9 vertices, $\Delta(G)=3$ and $\delta(G) \geq 2$. $G$ is 3 -separable with $(1,1)$ vertices.

Proof. It is easy to see that $G$ cannot be 3 -regular, so there is at least 1 vertex of degree 2 , let us denote it by $x$. Let us denote the two neighbors of $x$ by $u$ and $v$. If $u$ or $v$ have a neighbor of degree 3, let us denote it by $y . G \backslash M(y)$ has 5 vertices and $x$ is of degree 0 or 1 so that graph is 3 -separable by the $(0,1)$ vertices. On the other hand, if neither $u$ nor $v$ have a neighbor of degree 3 then at least 1 of them has a neighbor of degree 2 , not in $\{u, x, v\}$ and we distinguish three subcases:

1) One of them, say $u$, has a neighbor $y$ of degree 2 and $v$ is adjacent only to vertices in $\{x, M(y) \backslash\{y\}\}$.

Let us denote the other neighbor of $y$ by $z$. Now $G \backslash M(y)$ has 6 vertices, one component contains vertices $x$ and $v$ and the graph of 4 remaining vertices is 3separable with $(0,1)$ vertices.

2) One of them, say $u$, has a neighbor $y$ of degree 2 and $v$ is adjacent only to $x$ and $y$. This means that $u$ must have another neighbor, say $z$, of degree 2. Graph $G \backslash M(z)$ has 6 vertices, and one component contains only vertices $x, v$ and $y$ so the claim easily follows.

3) Both of $u$ and $v$ have neighbors, say $y$ and $w$ of degree 2, and $y$ has another neighbor, $z$ (not necessarily different from $w$ ).

If $w$ has a neighbor in $\{u, y, z\}$ then let us observe $G \backslash M(y)$.

3.1.) $v$ has the degree 2 in $G$. Now $G \backslash M(y)$ has 6 vertices, $x$ and $v$ form one component and the remaining graph of 4 vertices is 3 -separable by $(0,1)$ vertices. 
3.2.) $v$ has the degree 3 in $G$. Let us denote the neighbor of $v$ different from $x$ and $w$ by $v_{1}$. Now $G \backslash\left(M(y) \cup\left\{v_{1}\right\}\right)$ has all the components with at most 3 vertices.

On the other hand, if non $v$ neighbor of $w$ is not in $\{u, y, z\}$ then $G \backslash(M(y) \cup\{v\})$ has 5 vertices in two components so the claim again stands.

If $G$ is not connected it is easy to see that the claim also holds.

Lemma 4. Let $k \leq 5$ and let it hold that no three vertices can have all the colors. Then:

i) A graph $G$ with at most 6 vertices doesn't allow a highly $(0,1)$-resistant $k$ multicoloring.

ii) A graph $G$ with at most 7 vertices doesn't allow a highly (1,0)-resistant $k$ multicoloring.

iii) A graph $G$ with at most 10 vertices doesn't allow a highly $(1,1)$-resistant $k$-multicoloring.

iv) A graph $G$ with at most 11 vertices doesn't allow a highly $(2,0)$-resistant $k$-multicoloring.

Proof. We will prove all the claims for connected graphs and the claims for disconnected graphs follow from there. Without the loss of generality we can assume $k=5$.

i) Let $G$ be a connected graph with at most 6 vertices. Each color must be assigned at least 2 times so at least one vertex will have at least two colors. Moreover, no vertex can have three or more colors because no three vertices can have all the colors. Let us assume that one of the vertices has the set of colors $\{1,2\}$. Now neither of the remaining vertices can have neither of the sets $\{3,4\},\{3,5\},\{4,5\}$. But then it is impossible to assign the colors two times each.

ii) Let $G$ be a connected graph with at most 7 vertices. If there is a vertex of degree at least 3 in $G$ the claim is easily seen, so let $\Delta(G)=2$. If $G$ is a path the claim can again be easily seen so let us assume $G$ is a cycle. No vertex can have 3 or more colors and every color must be assigned at least twice so at least one vertex must have two colors. Let us assume one of the vertices has the set of colors $\{1,2\}$ and let us denote that vertex by $u_{1}$. Further, let us denote the rest of the vertices in the cycle by $u_{2}, \ldots, u_{7}$, starting from $u_{1}$ in any direction. Now, no other vertex can have neither of the sets $\{3,4\},\{3,5\},\{4,5\}$, and since each of those colors must appear at least twice we assign the colors $3,4,5$ to the remaining 6 vertices, one to each. It is obvious that the colors 3,4,5 will not appear anywhere else so there is no point in assigning them in such a way that one color is given to two vertices that are on a distance 2 or less, since then both instances of that single color could be 
easily removed. So without the loss of generality we may assume the multicoloring function is the following:

$\begin{array}{ccccccc}u_{1} & u_{2} & u_{3} & u_{4} & u_{5} & u_{6} & u_{7} \\ 1,2 & 3 & 4 & 5 & 3 & 4 & 5\end{array}$

Now we must assign the colors 1 and 2 once more each. It is easy to see that if we assign the colors 1 and 2 to two vertices that have different colors, three vertices could be found that have all the colors, so the only option is to assign 1 and 2 only to $u_{2}$ and $u_{5}$, or $u_{3}$ and $u_{6}$, or $u_{4}$ and $u_{7}$, one to each. However in each of those possibilities a vertex can be chosen to remove both instances of one of the colors.

iii) Let $G$ be a connected graph with at most 10 vertices. Each color must be assigned at least 3 times so at least one vertex will have 2 or more colors. If one vertex would have 3 or more colors it would be easy to find three vertices that have all the colors, so let us assume that one of the vertices has exactly 2 keys, the set $\{1,2\}$. Now none of the other vertices can have neither of the sets $\{3,4\},\{3,5\}$, $\{4,5\}$ and since each of those colors must be assigned at least 3 times each, we must assign them to the remaining 9 vertices so that each vertex has exactly one color from the set $\{3,4,5\}$. The colors 1 and 2 must be assigned twice more each so obviously two of the 9 vertices that have different colors from $\{3,4,5\}$ will have different colors from $\{1,2\}$. But then again 3 vertices can be found that have all the colors.

iv) Let $G$ be a connected graph with at most 11 vertices. If there is a vertex of degree 3 in $G$ then let us denote it by $u$. $G \backslash M(u)$ has 7 vertices and the claim follows from ii). Let us assume that the highest degree in $G$ is 2 . If $G$ is a path the claim is easy to see so let us assume $G$ is a cycle. Let $u$ be any vertex in $G$. $G \backslash M(u)$ is a path of 8 vertices and by denoting one of the central vertices with $v$ we can see that $G \backslash(M(u) \cup M(v))$ has all the components of size at most 3.

Lemma 5. Let $k \leq 5$ and let it hold that no three vertices can have all the colors. Then:

i) A graph $G$ with 7 vertices that is either a path or it is disconnected doesn't allow a highly $(0,1)$-resistant $k$-multicoloring.

ii) A graph $G$ with 11 vertices that is either disconnected or it has a minimal degree 1 doesn't allow a highly $(1,1)$-resistant $k$-multicoloring.

iii) A disconnected graph $G$ with 15 vertices doesn't allow a highly $(2,1)$-resistant $k$-multicoloring. 
Proof. i) If $G$ is a disconnected graph with 7 vertices the claim follows from Lemma $4 \mathrm{i})$, and if $G$ is a path it is 3 -separable with $(0,1)$ vertices.

ii) Let $G$ be a disconnected graph with 11 vertices. The claim follows from Lemma 4 i), ii) and iii). Now let $G$ be a connected graph with 11 vertices such that $\delta(G)=1$. Let us denote with $x$ a vertex of degree 1 and with $y$ its only neighbor. We distinguish two cases.

1) $y$ has the degree 2 . We follow the path starting in $y$ until we reach a vertex of degree 3. If such a vertex doesn't exist $G$ is a path and it is 3 -separable with $(1,1)$ vertices. On the other hand, if such a vertex exists, let us denote it by $u$. $G \backslash M(u)$ has 7 vertices and it is either disconnected or it is a path so the claim follows from i).

2) $y$ has the degree 3 . If any neighbor of $y$ has the degree 3 let us denote it by $u . G \backslash M(u)$ is disconnected ( $x$ is isolated) with 7 vertices so the claim follows from i). Let us assume both neighbors of $y$ have the degree at most 2. If they have a common neighbor other then $y$, it must have the degree 3 so by removing it along with its neighbors we again obtain a disconnected graph with 7 vertices and the claim follows as before. If one of those neighbors has the degree 1 then let us observe the other neighbor of $y$, of degree 2. By following the path starting in that neighbor, not containing $y$, let us denote by $w$ the first vertex of degree 3 we find. If $G \backslash M(w)$ is disconnected the claim follows from i), and if it is connected then $G \backslash M(w)$ is a path of 5 vertices with two leaves attached to one end of it (vertex $y$ ). If we denote by $z$ the neighbor of $y$ that has the degree 2 , then $G$ is 3 -separable by $w$ and $z$.

Let us assume both neighbors of $y$ have the degree 2 and each has its own neighbor. Let us denote those neighbors by $u$ and $v$. We consider three subcases.

2.1.) $u$ and $v$ are adjacent. In that case at least one of them must have the degree 3 , without the loss of generality let us assume $d(u)=3 . G \backslash M(u)$ is disconnected with 7 vertices so the claim follows from i).

2.2.) $u$ and $v$ are not adjacent and at least one of them has the degree 3 . We may assume $d(u)=3$. Now $G \backslash(M(u) \cup\{v\})$ has all the components with at most 3 vertices.

2.3.) $u$ and $v$ both have the degree 2. If they have a common neighbor it must have the degree 3 , so the claim follows as before. Let us assume each of them has its own neighbor, let us denote them by $u_{1}$ and $v_{1}$, respectively. If $u_{1}$ and $v_{1}$ are adjacent the claim follows as in 2.1. and if they are not adjacent and at least one of them has the degree 3 then it is easy to see that $G$ is 3 -separable, similarly as in 2.2. Let us assume $u_{1}$ and $v_{1}$ both have the degree 2 , and let us denote their 
neighbors by $u_{2}$ and $v_{2}$, respectively. If $u_{2}$ and $v_{2}$ are adjacent, at least one of them must have the degree 3 , without the loss of generality we may assume $d\left(u_{2}\right)=3$. Now $G \backslash\left(M\left(u_{2}\right) \cup\{y\}\right)$ has all the components with at most 3 vertices. If any of $u_{2}$, $v_{2}$ has the degree 1 then $G \backslash M(y)$ has 7 vertices and is disconnected so the claim follows from i). And if they are not adjacent and both have the degree 2, they are both adjacent to the 1 remaining vertex in the graph. But now $G \backslash M(y)$ is a path of 7 vertices and the claim again follows from i).

iii) Let $G$ be a graph with 15 vertices and at least 2 components.

If the smallest component in $G$ has 1 vertex it obviously cannot have all the colors so let us observe the remaining 14 vertices. If there exist a vertex of degree 3 among those 14 vertices let us denote it by $u$. $G \backslash M(u)$ a graph with 10 vertices which is doesn't allow a highly $(1,1)$-resistant $k$-multicoloring by Lemma 4 iii). The same reasoning follows if the smallest component in $G$ has 2 or 3 vertices.

If the smallest component in $G$ has 4 vertices then that component is 3-separable with $(0,1)$ vertices and the graph remaining 11 vertices doesn't allow a highly $(2,0)$ resistant $k$-multicoloring by Lemma 4 iv).

If the smallest component in $G$ has 5,6 or 7 vertices then by Lemma 4 ii) it doesn't allow a highly $(1,0)$-resistant $k$-multicoloring and the graph of remaining 10,9 or 8 vertices doesn't allow a highly $(1,1)$-resistant $k$-multicoloring by Lemma 4 iii).

Lemma 6. Let $G$ be a connected graph with 19 vertices, $\Delta(G)=3$, and $\Delta(G \backslash M(u))=$ 3 , for any vertex $u$ with degree 3 in $G$. Then one of the following holds:

a) There exists a vertex $u$ in $G$ such that $G \backslash M(u)$ is disconnected with 15 vertices.

b) There are vertices $u$ and $v$ in $G$ such that $G \backslash(M(u) \cup M(v))$ has 11 vertices and it is either disconnected or it has the minimal degree 1.

Proof. We will prove the claim through two cases, depending on the minimal degree in $G$. Obviously $G$ cannot be 3 -regular so its minimal degree is either 1 or 2 .

1) $\delta(G)=1$. Let us denote by $x$ the vertex of degree 1 and by $y$ its only neighbor. If $y$ has the degree 2 we follow the path starting in $y$, not containing $x$, and denote by $u$ the first vertex of degree 3 in that path. $G \backslash M(u)$ has 15 vertices and it is disconnected, so a) holds. Let us assume $y$ has the degree 3 and let us denote the other two neighbors of $y$ by $u$ and $v$. If any of them has the degree 3 then by removing it and its neighbors, we obtain a disconnected graph ( $x$ is isolated) with 15 vertices and again a) holds. If any of $u$ and $v$ has the degree 1 the claim is easy to see, similarly as when $y$ has the degree 2 . So let us assume $u$ and $v$ both 
have the degree 2. If $u$ and $v$ have a common neighbor it must have the degree 3 so by removing and its neighbors, we again obtain a disconnected graph with 15 vertices. Let us assume $u$ and $v$ have each its own new neighbor and let us denote them by $u_{1}$ and $v_{1}$, respectively. If any of them has the degree 3 , without the loss of generality we may assume that $d\left(u_{1}\right)=3$, then $y$ has the degree 2 in $G \backslash M\left(u_{1}\right)$ so when we remove another vertex of degree 3 from $G \backslash M\left(u_{1}\right)$ along with its neighbors, the remaining graph will have 11 vertices and $x$ will have the degree 1 in it, so b) holds. If any of $u_{1}$ and $v_{1}$ has the degree 1 , let us assume $d\left(u_{1}\right)=1$, then whatever two vertices of degree 3 we remove along with their neighbors, $u_{1}$ will have the degree 0 or 1 in the remaining graph of 11 vertices so b) holds. On the other hand, if $u_{1}$ and $v_{1}$ both have the degree 2 we observe the rest of the graph. There must be at least one vertex besides $y$ with degree 3 . Let us remove it and its neighbors. Now by removing $y$ and its neighbors at least one of $u_{1}$ and $v_{1}$ remains either isolated or with the degree 1 in the remaining graph of 11 vertices, so again b) holds.

2) $\delta(G)=2$. Let us distinguish two subcases.

2.1.) Two vertices of degree 2 are adjacent in $G$.

Let us denote those vertices by $x$ and $y$. If $x$ and $y$ have a common neighbor of degree 3 let us denote it by $u$ and let us observe the path starting in $u$ not containing $x$ nor $y$. Let us denote by $w$ the first vertex of degree 3 on that path. If $G \backslash M(w)$ is disconnected then a) holds and if it is connected then $u$ is the only vertex of degree 3 in the remaining graph and by removing it and its neighbors, we obtain a path of 11 vertices so b) holds. Let us assume $x$ and $y$ have one more neighbor each. If they are adjacent at least one of them must have the degree 3 and by removing it and its neighbors, either $x$ or $y$ will remain isolated so a) holds. Let us assume the neighbors are not adjacent and let us denote them by $x_{1}$ and $y_{1}$. If at least one of those neighbors has the degree 2 , without the loss of generality we may assume that it is $x_{1}$, then let us follow the path starting in $x$, not passing through $y$, and let us denote by $u$ the first vertex of degree 3 in that path (not necessarily different from $y_{1}$ ) (such a vertex must exist because $\delta(G)>1$ ), and let us denote the predecessor of the predecessor of $u$ by $v$ (note that $v$ can be $x$ if we found $u$ on a distance 2 from $x)$. Now vertex $v$ has the degree 1 in $G \backslash M(u)$ and since its only neighbor is surely of degree 2 then by removing the other vertex of degree 3 and its neighbors (one more vertex of degree 3 must exist) from $G \backslash M(u), v$ will remain with the degree at most 1 and b) holds.

So let us assume that both neighbors, of $x$ and $y$, have the degree 3 . Let us again denote the neighbors by $x_{1}$ and $y_{1}$, respectively. If they have a common neighbor then let us remove $x_{1}$ and its neighbors. Now $y$ has the degree 1 and $y_{1}$ has the 
degree 2 in $G \backslash M\left(x_{1}\right)$ so after removing the other vertex of degree 3 and its neighbors (one more vertex of degree 3 must exist), $y$ will have the degree at most 1 and b) holds.

Let us assume $x_{1}$ and $y_{1}$ have no common neighbors but instead have 2 more neighbors each. If any of them has the degree 3, without the loss of generality we may assume that it is the neighbor of $x_{1}$, then by removing it and its neighbors, $x$ remains with the degree 1 and it will have the degree at most 1 after removing the other vertex of degree 3 along with its neighbors, by the same reasoning as before. Let us assume that all the neighbors of $x_{1}$ and $y_{1}$ have the degree 2 .

If two of them are adjacent, both of them from $x_{1}$ (or $y_{1}$ ), then it is easy to see that 1 vertex of degree 3 can be found to leave the graph disconnected with 15 vertices. And if one neighbor of $x_{1}$ is adjacent to a neighbor of $y_{1}$ then we observe the other neighbors of $x_{1}$ and $y_{1}$ and let us denote them by $x_{2}$ and $y_{2}$, respectively. They obviously cannot be adjacent and if they have a common neighbor it must be of degree 3, so by removing it and its neighbors, we obtain a disconnected graph with 15 vertices. Let us assume $x_{2}$ and $y_{2}$ have each its own neighbor and let us denote them by $x_{3}$ and $y_{3}$, respectively. If any of $x_{3}$ and $y_{3}$ has the degree 3 , without the loss of generality let us assume it is $x_{3}$, then $G \backslash\left(M\left(x_{3}\right) \cup M\left(y_{1}\right)\right)$ is disconnected with 11 vertices so b) holds. Let us assume $x_{3}$ and $y_{3}$ have the degree 2 . Now if we remove $x_{1}$ and its neighbors, $y$ and $x_{3}$ have the degree 1 in $G \backslash M\left(x_{1}\right)$. If we remove $y_{1}$ and its neighbors, then $x_{3}$ will have the degree 1 in the remaining graph of 11 vertices and otherwise $y$ will remain with degree 1 so in each case a) holds.

On the other hand, if none of the neighbors of $x_{1}$ and $y_{1}$ are adjacent let us denote them by $x_{11}, x_{12}, y_{11}, y_{12}$. If $x_{11}$ and $x_{12}$ (or $y_{11}$ and $y_{12}$ ) have a common neighbor of degree 2 or 3 it is easy to obtain a disconnected graph. Let us assume that some other two have a common neighbor and without the loss of generality let us assume those are $x_{12}$ and $y_{11}$. (Figure 1 a) If that neighbor has the degree 2 then $G \backslash\left(M\left(x_{1}\right) \cup M\left(y_{1}\right)\right)$ is disconnected with 11 vertices and if it has the degree 3 then that vertex will have the degree at most 1 in $G \backslash\left(M\left(x_{1}\right) \cup M\left(y_{1}\right)\right)$ so b) holds. Let us assume none of $x_{11}, x_{12}, y_{11}, y_{12}$ have a common neighbor and let us denote their neighbors by $x_{21}, x_{22}, y_{21}, y_{22}$ (Figure $\left.1 \mathrm{~b}\right)$ ).

If any of $x_{21}, x_{22}, y_{21}, y_{22}$ has the degree 3 , without the loss of generality let us assume $x_{21}$ then $G \backslash\left(M\left(x_{21}\right) \cup M\left(y_{1}\right)\right)$ has 11 vertices and minimal degree 1 $(d(x)=1)$, so let us assume they all have the degree 2. But now $y, x_{21}$ and $x_{22}$ have the degree 1 in $G \backslash M\left(x_{1}\right)$ and when we remove any vertex of degree 3 and its neighbors, at least one of them will have the degree at most 1 in the remaining graph of 11 vertices. 


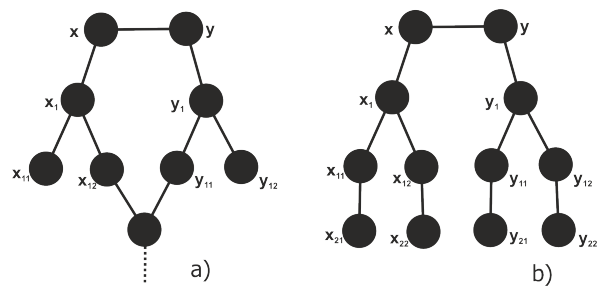

Figure 1: Subcases of 2.1.

2.2.) There are no adjacent vertices of degree 2 .

Let $x$ be a vertex of degree 2 and $u$ and $v$ its neighbors of degree 3 . Let us consider two subcases.

2.2.1.) $u$ and $v$ are adjacent.

If they have a common neighbor it must have the degree 3 and by removing it and its neighbors, we obtain a disconnected graph with 15 vertices.

If $u$ and $v$ have one more neighbor each and at least one of them has the degree 3 , without the loss of generality let us assume it is the neighbor of $u$, then by removing it and its neighbors, $x$ remains with degree 1 and $v$ with degree 2 , so by removing the other vertex of degree 3 and its neighbors from the graph the degree of $x$ will be at most 1 and b) will hold. Let us assume both neighbors have the degree 2 and let us denote them by $u_{1}$ and $v_{1}$. If $u_{1}$ and $v_{1}$ have a common neighbor the claim is again easy to see so let us assume they have one more neighbor each and let us denote them by $u_{2}$ and $v_{2}$. They must have the degree 3 because there are no adjacent vertices of degree 2 in the graph.

If $u_{2}$ and $v_{2}$ are adjacent then by removing any of them and its neighbors, we obtain a disconnected graph on 15 vertices and if they have a common neighbor of degree 3 then by removing it and its neighbors, we obtain a disconnected graph on 15 vertices. If $u_{2}$ and $v_{2}$ have a common neighbor of degree 2 then $G \backslash\left(M\left(u_{2}\right) \cup M(v)\right)$ has 11 vertices and $v_{2}$ has the degree at most 1 so b) holds. And if they have no common neighbors then $G \backslash\left(M\left(u_{2}\right) \cup M\left(v_{2}\right)\right)$ is disconnected with 11 vertices.

2.2.2.) $u$ and $v$ are not adjacent.

If they have a common neighbor of degree 3 the claim is easy to see. Let us first assume they have a common neighbor of degree 2 and let us denote their other neighbors by $u_{1}$ and $v_{1}$ (they cannot have 2 common neighbors of degree 2 ). If at least one of them has the degree 3, without the loss of generality let us assume that it is $u_{1}$, then in $G \backslash M\left(u_{1}\right) x$ has the degree 1 . If $v$ is not the only vertex of degree 3 in 
$G \backslash M\left(u_{1}\right)$ then by removing that other vertex we obtain a graph with 11 vertices in which $x$ has the degree 1 . And if $v$ is the only vertex with the degree 3 in $G \backslash M\left(u_{1}\right)$ then that means $v_{1}$ has the degree at most 2 in $G \backslash M\left(u_{1}\right)$ and by removing $v$ and its neighbors, the other (non $v$ ) neighbor of $v_{1}$ remains with the degree 1 in a graph of 11 vertices or we have obtained a disconnected graph. So let us assume that $u_{1}$ and $v_{1}$ have the degree 2 . They are obviously not adjacent and if they have a common neighbor of degree 3 the claim is easily seen, so let us assume they each have a new neighbor of degree 3 , let us denote them by $u_{2}$ and $v_{2}$. Now $G \backslash\left(M\left(u_{2}\right) \cup M(v)\right)$ is disconnected ( $u$ is isolated) with 11 vertices.

If $u$ and $v$ have no common neighbors let us denote their neighbors by $u_{1}, u_{2}$, $v_{1}, v_{2}$. If any of them has the degree 3 , without the loss of generality let us assume it is $u_{1}$, then $x$ has the degree 1 in $G \backslash M\left(u_{1}\right)$. If there exists a vertex of degree 3 in $G \backslash M\left(u_{1}\right)$ different from $v$ then by removing it as the other agent $x$ has the degree at most 1 in the remaining graph of 11 vertices. And if $v$ is the only vertex with degree 3 in $G \backslash M\left(u_{1}\right)$ then $v_{1}$ and $v_{2}$ have the degree at most 2 in $G \backslash M\left(u_{1}\right)$ and by removing $v$ and its neighbors we either obtain a disconnected graph or at least one neighbor of $v_{1}$ and $v_{2}$ has the degree at most 1 in $G \backslash\left(M\left(u_{1}\right) \cup M(v)\right)$. The only remaining case to consider is when $u_{1}, u_{2}, v_{1}, v_{2}$ all have the degree 2 . Neither two of them may be adjacent and they cannot have common neighbors of degree 2 . If any two of them have a common neighbor of degree 3 the claim is easy to see and if they have no common neighbors then let us remove the neighbor of $u_{1}$ and its neighbors, and $M(v)$. The remaining graph has 11 vertices and $u$ has the degree 1 so the claim is proven.

Theorem 7. 1. A graph $G$ with at most 17 vertices doesn't allow a highly $(3,1)$ resistant multicoloring.

2. $6 \leq H R_{3,1}(19) \leq H R_{3,1}(18) \leq 7$.

3. $H R_{3,1}(20)=4$.

Proof. First we observe that that if $G$ allows a highly $(3,1)$-resistant $k$-multicoloring, then $k \geq 4$. Let us first prove that a graph $G$ with at most 17 vertices doesn't allow a highly $(3,1)$-resistant multicoloring. Let us assume the opposite, that there exists a graph $G$ with 17 vertices and $k \in \mathbb{N}$, such that $G$ allows a highly $(3,1)$-resistant $k$-multicoloring. Components of at most 3 vertices cannot have all the colors so we disregard them. We will prove the claim through four cases, depending on the number of components with at least 4 vertices in $G$.

1) $G$ has 4 components with at least 4 vertices. 
The largest component in $G$ has at most 5 vertices and it is 3 -separable with $(1,0)$ vertices. The second and third largest component are also 3-separable with $(1,0)$ vertices, and the fourth largest component is 3 -separable with $(0,1)$ vertices. This means that $G$ is 3 -separable by $(3,1)$ vertices and therefore doesn't allow the aforementioned coloring.

2) $G$ has 3 components with at least 4 vertices.

If the largest component in $G$ has 9 or 8 vertices then it is 3 -separable with $(2,0)$ vertices by Lemma 1 ii), the second largest component is 3 -separable with $(1,0)$ vertices and the remaining component with $(0,1)$ vertices.

If the largest component in $G$ has 7 vertices it is 3 -separable with $(1,1)$ vertices by Lemma $1 \mathrm{i}$ ) and the two remaining components are 3 -separable by $(1,0)$ vertices each.

3) $G$ has 2 components with at least 4 vertices.

If the largest component in $G$ has 13 vertices it is 3 -separable with $(3,0)$ vertices by Lemma $1 \mathrm{iv}$ ), and the remaining component of at most 4 vertices is 3 -separable with $(0,1)$ vertices.

If the largest component in $G$ has 11 or 12 vertices it is 3 -separable with $(2,1)$ vertices by Lemma 1 iii) and it is easily seen that the remaining component is 3 -separable with $(1,0)$ vertices.

If the largest component has 9 or 10 vertices it is 3 -separable with $(2,0)$ vertices by Lemma $1 \mathrm{ii}$ ) and the remaining component is 3 -separable with $(1,1)$ vertices by Lemma 1 i).

4) $G$ has exactly 1 component with at least 4 vertices.

Let $G$ be a connected graph with 17 vertices. We can assume this because all other cases are implied by this solution. We consider 3 subcases, depending on the highest degree in $G$.

4.1.) The highest degree in $G$ is 4 .

Let $u$ be a vertex of degree 4 in $G$. $G \backslash M(u)$ has 12 vertices and it is 3 -separable with $(2,1)$ vertices by Lemma 1 iii).

4.2.) The highest degree in $G$ is 2 .

$G$ is obviously a cycle or a path and by choosing a vertex $u$ of degree $2, G \backslash M(u)$ is a union of paths and has 14 vertices. It can now be easily seen that the claim holds.

4.3.) The highest degree in $G$ is 3 .

Let us denote any vertex of degree 3 by $u$. $G \backslash M(u)$ has 13 vertices. If the highest degree in $G \backslash M(u)$ is at most 2 the claim easily follows so let us assume the highest degree in $G \backslash M(u)$ is 3. We distinguish 2 possibilities. 
a) There is a vertex of degree 1 in $G \backslash M(u)$. The claim now follows from Lemma 2 ii).

b) The minimal degree in $G \backslash M(u)$ is 2 . Let $v$ be a vertex in $G \backslash M(u)$ with degree 3. $G \backslash(M(u) \cup M(v))$ has 9 vertices. If the highest degree in $G \backslash(M(u) \cup M(v))$ is at most 2 the claim can easily be seen, so let us assume the highest degree in $G \backslash(M(u) \cup M(v))$ is 3. Also, if there exists a vertex of degree 1 in $G \backslash(M(u) \cup M(v))$, the claim follows from Lemma $2 \mathrm{i})$. The only case left to consider is if all the vertices in $G \backslash(M(u) \cup M(v))$ are of degree 2 or 3 . But in this case the claim follows from Lemma 3. We have proven that a graph $G$ with at most 17 vertices doesn't allow a highly $(3,1)$-resistant multicoloring.

A graph with 18 vertices that that allows a highly $(3,1)$-resistant 7 -multicoloring is given in Figure 2.
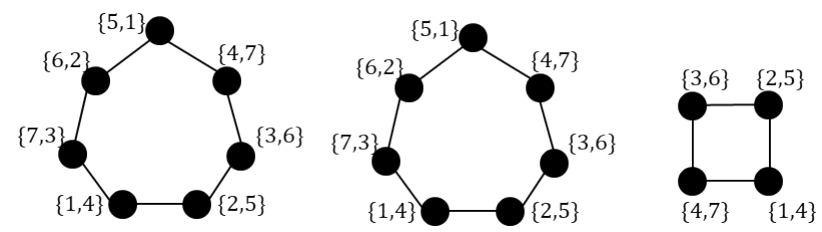

Figure 2: A graph with 18 vertices and a highly $(3,1)$-resistant 7multicoloring

From this it follows that $H R_{3,1}(19) \leq 7$.

Let us prove that $H R_{3,1}(19) \geq 6$. Let us assume the opposite, that there exists a graph $G$ with 19 vertices that allows a highly $(3,1)$-resistant 5 -multicoloring.

Since no three vertices can have all the colors it is enough to observe only components with 4 or more vertices. We distinguish four possibilities:

1) $G$ has four components with at least 4 vertices.

The largest component has at most 7 vertices so by Lemma 4 ii) it doesn't allow a highly $(1,0)$-resistant 5-multicoloring. The second and third largest component also don't allow a highly (1,0)-resistant 5-multicoloring and the smallest of the 4 observed components can have 4 vertices at most so it is 3 -separable by $(0,1)$ vertices.

2) $G$ has three components with at least 4 vertices.

Possible component sizes are different, depending on how many vertices in $G$ are in components with less then 4 vertices. However, the largest component can have 11 vertices at most so by Lemma 4 iv) it doesn't allow a highly $(2,0)$-resistant 
5-multicoloring. Second largest component can have 7 vertices at most so by Lemma 4 ii) it doesn't allow a highly $(1,0)$-resistant 5 -multicoloring and the third largest component has at most 6 vertices so by Lemma 4 i) it doesn't allow a highly $(0,1)$ resistant 5-multicoloring. This holds for all possible sizes of the components.

3) $G$ has two components with at least 4 vertices.

The largest component has 15 vertices at most and the fact that a graph of 15 vertices doesn't allow a highly $(3,0)$-resistant 5 -multicoloring follows from the results in [7]. The second largest component has 4 vertices and it is 3 -separable by $(0,1)$ vertices. The case when the largest component has 14 or 13 vertices and the second largest 5 or 6 follows in the same way.

If the largest component has 12 vertices then it is 3 -separable by $(2,1)$ vertices which follows from Lemma 1 iii). The second largest component has at most 7 vertices and it doesn't allow a highly $(1,0)$-resistant 5 -multicoloring, by Lemma 4 ii).

If the largest component has 11 or 10 vertices then it doesn't allow a highly $(2,0)$-resistant 5 -multicoloring and the second largest component then has at most 8 or 9 vertices and it doesn't allow a highly $(1,1)$-resistant 5 -multicoloring, which follows from Lemma 4 iv) and iii).

4) $G$ has exactly one component with at least 4 vertices.

Without the loss of generality we may assume that $G$ is connected with 19 vertices because all other cases are implied by this solution.

4.1.) The highest degree in $G$ is 2 . Let $u$ be any vertex with degree 2 . $G \backslash M(u)$ has 16 vertices and it is a union of paths. Let us denote by $v$ the center of the largest path in $G \backslash M(u)$. Now $G \backslash(M(u) \cup M(v))$ has 13 vertices and it is a union of at least 2 paths of which the largest one has at most 7 vertices and it is doesn't allow a highly $(1,0)$-resistant 5 -multicoloring by Lemma 4 ii) The remaining grapf of 6 vertices doesn't allow a highly $(0,1)$-resistant 5 -multicoloring by Lemma 4 i).

4.2.) The highest degree in $G$ is 3 . Let $u$ be any vertex with degree 3 . $G \backslash M(u)$ has 15 vertices.

If all of them have the degree at most 2 then let $v$ be any vertex of degree 2 . $G \backslash(M(u) \cup M(v))$ has 12 vertices and it is a union of paths. If $G \backslash(M(u) \cup M(v))$ is not connected it is easy to see that it is 3 -separable with $(1,1)$ vertices and if it is one path of 12 vertices then let $w$ be a vertex on a distance 4 from the end of that path. $G \backslash(M(u) \cup M(v) \cup M(w))$ is a union of two paths, of lengths three and six, and the larger one is 3 -separable with $(0,1)$ vertices.

On the other hand, if there exists a vertex of degree 3 in $G \backslash M(u)$ than the claim follows from Lemma 6 and Lemma 5 ii) and iii). 
4.3.) The highest degree in $G$ is at least 4 . Let $u$ be a vertex in $G$ with the highest degree. $G \backslash M(u)$ has at most 14 vertices and it doesn't allow a highly $(2,1)$-resistant 5 -multicoloring by Lemma 5 iii). This proves our claim that $H R_{3,1}(19) \geq 6$.

It remains to prove that $H R_{3,1}(20)=4$. It is easily seen that $H R_{3,1}(20) \geq 4$ must hold, and the graph $G$ with 20 vertices that allows a highly $(3,1)$-resistant 4-multicoloring is given in Figure 3.

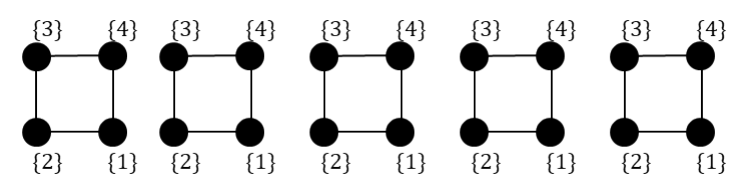

Figure 3: A graph with 20 vertices and a highly (3,1)-resistant 4multicoloring

\section{Acknowledgements}

Partial support of the Croatian Ministry of Science and Education is gratefully acknowledged.

\section{References}

[1] B. Bollobas, Modern Graph Theory, Springer, New York, 1998.

[2] Halldórsson, M. M. and Kortsarz, G. (2002). Tools for multicoloring with applications to planar graphs and partial k-trees. Journal of Algorithms, 42(2), 334-366.

[3] Halldórsson, M. M., and Kortsarz, G. (2004, August). Multicoloring: Problems and techniques. In International Symposium on Mathematical Foundations of Computer Science, pp. 25-41.

[4] Harary, F. (1985). Conditional colorability in graphs. Graphs and applications (Boulder, Colo., 1982), Wiley-Intersci. Publ, 127-136. 
[5] Marx, D. (2004). Graph colouring problems and their applications in scheduling. Periodica Polytechnica Electrical Engineering, 48(1-2), 11-16.

[6] Shamir, A. (1979) How to share a secret. Communications of the ACM 22, 11, 612-613.

[7] Vojković, T., Vukičević, D. and Zlatić, V. (2018). Multicoloring of Graphs to Secure a Secret. Rad HAZU, Matematičke znanosti, 22, 1-22.

[8] Vojković, T. and Vukičević, D. (2018). Highly $(a, b)$-resistant multicoloring of graphs. Submitted to Annales Mathematicae et Informaticae.

[9] Zhang, Z., Liu, L. and Wang, J. (2002). Adjacent strong edge coloring of graphs. Applied Mathematics Letters, 15(5), 623-626. 